\title{
Delphi Survey for Designing a Intervention Research Study on Childhood Obesity Prevention
}

\author{
Min Jeong Kim ${ }^{1}$, Eunju Sung ${ }^{2, *}$, Eun Young Choi', Young-Su Ju ${ }^{3}$ Eal-Whan Park', Yoo-Seock Cheong', Sunmi Yoo ${ }^{4}$, \\ Kyung Hee Park ${ }^{5}$, Hyung Jin $\mathrm{Choi}^{6}$, Seolhye Kim² \\ 'Department of Family Medicine, Dankook University College of Medicine, Cheonan, Korea \\ ${ }^{2}$ Department of Family Medicine, Kangbuk Samsung Hospital, Sungkyunkwan University School of Medicine, Seoul, Korea \\ ${ }^{3}$ Department of Occupational and Environmental Medicine, Hallym University Sacred Heart Hospital, Anyang, Korea \\ ${ }^{4}$ Department of Family Medicine, Inje University Haeundae Paik Hospital, Busan, Korea \\ ${ }^{5}$ Department of Family Medicine, Hallym University Sacred Heart Hospital, Anyang, Korea \\ ${ }^{6}$ Department of Anatomy, Seoul National University College of Medicine, Seoul, Korea
}

Background: The prevalence of childhood obesity in South Korea has increased owing to economic improvement and the prevailing Westernized dietary pattern. As the incidence of chronic diseases caused by obesity is also expected to increase, effective interventions to prevent childhood obesity are needed. Therefore, we conducted a Delphi study to determine the priorities of a potential intervention research on childhood obesity prevention and its adequacy and feasibility.

Methods: The two-round Delphi technique was used with a panel of 10 childhood obesity experts. The panelists were asked to rate "priority populations," "methods of intervention," "measurement of outcomes," "future intervention settings," and "duration of intervention" by using a structured questionnaire. Finally, a portfolio analysis was performed with the adequacy and feasibility indexes as the two axes.

Results: For priority populations, the panel favored "elementary," "preschool," and "middle and high school" students in this order. Regarding intervention settings, the panelists assigned high adequacy and feasibility to "childcare centers" and "home" for preschool children, "school" and "home" for elementary school children, and "school" for adolescents in middle and high school. As the age of the target population increased, the panelists scored increasing numbers of anthropometric, clinical, and intermediate outcomes as highly adequate and feasible for assessing the effectiveness of the intervention.

Conclusion: According to the results of the Delphi survey, the highest-priority population for the research on childhood obesity prevention was that of elementary school students. Various settings, methods, outcome measures, and durations for the different age groups were also suggested.

Keywords: Delphi Technique; Intervention; Prevention; Obesity; Childhood 


\section{INTRODUCTION}

According to the World Health Organization, the global prevalence of obesity doubled from 1980 to 2014, and the prevalence of childhood obesity also increased owing to improved living standards and a greater preference for the Westernized diet. ${ }^{1)}$ An increasing childhood obesity rate has become a global trend and is emerging as a serious public health issue. ${ }^{2)}$ In South Korea, the prevalence of childhood obesity increased from $14.3 \%$ in 2010 to $15.3 \%$ in $2013 .{ }^{3)}$

Childhood obesity confers a burden on society by engendering chronic physical diseases and psychosocial problems. ${ }^{4-6)}$ According to data from the Korea Institute for Health and Social Affairs, the annual socioeconomic cost of childhood obesity has reached approximately 1.3638 trillion won. ${ }^{7)}$ In addition, $80 \%$ of obese children become obese adults, which increases their risk of chronic diseases and complications in adulthood, thereby increasing the burden of medical costs in subsequent generations. ${ }^{8,9)}$ Therefore, effective management of childhood obesity will help to reduce the socioeconomic burden arising from obesity.

Although a diverse range of school- and community-based intervention programs is being implemented to prevent and manage childhood obesity in Korea, ${ }^{10-14)}$ well-designed, evidence-based intervention trials are scarce. Most Korean intervention trials for prevention of childhood obesity were small-scale and short-term, without significant effects, and targeted only obese children rather than the entire child population. ${ }^{11-13)}$ In addition, the assessment methods used in the interventions or contents thereof were rarely evidence based, with most of them being selected and used arbitrarily by the researchers. ${ }^{10,14)} \mathrm{Na}-$ tionwide, non-profit support is needed to boost prevention research. Most of the national research funding for obesity prevention has been short-term and insufficient for a specific purpose. This might be the main reason for the lack of prevention program trials in Korea.

This study aimed to gather the opinions of specialists about the effective methods in designing of a research on childhood obesity intervention in Korea by using the Delphi technique. ${ }^{15,16)}$

\section{METHODS}

\section{Selection of the Panel}

The panel selection was decided by the research team and confirmed by consultation. The inclusion criteria were as follow: First, a broad range of experts were selected among clinicians, nurses, clinical nutritionists, and researchers in preventive medicine, nutrition, exercise, school health, or policies on childhood obesity prevention. Second, the expert should practice or be involved in a research project for childhood obesity prevention as a person in charge for $>1$ year. Third, the panel should consist of experts who represent a broad range of expertise and perspectives as related to the childhood obesity prevention program. The final members of the panel were 10 experts who consented to participate in the study.

\section{Questionnaire Survey}

We selected the final issues for discussion after reviewing domestic and international literatures on childhood obesity interventions, brainstorming within the research group, and discussing the matter with specialists in childhood obesity. ${ }^{17-20)}$ This corresponds to a modified Delphi technique, as the traditional Delphi technique uses an open-type questionnaire.

A questionnaire was developed to assess the priority population, and the adequacy and feasibility of various items necessary for designing the research on childhood obesity prevention. The questionnaire consisted of five assessment areas, which were "priority populations," "methods of intervention," "settings of intervention," "measurement of outcomes," and "duration of intervention." For the questions about the priority population of the intervention, the panel members rated the value of addressing each item from $1-5$, where 1 is the highest value (score of 5) and 5 is the lowest value (score of 1 ). The scores for each of the questions were summed to compute the score for the level of importance. The survey also contained questions that addressed the adequacy of 3 representative intervention methods, and the anticipated effect size and feasibility of 4 intervention settings. In addition, the panelists were asked to evaluate the adequacy and feasibility of key variables for assessing intervention outcomes, including 7 anthropometric indexes, 5 clinical indexes, and 6 intermediate variables. Finally, the survey included questions about whether 5 potential intervention durations would be appropriate for assessing the intervention effects. A 5-point scale was applied to each assessment question, with scores of 1 or 2 defined as negative; 3 , as having reservations; and 4 or 5 as affirmative. Prior to the commencement of the survey, the questionnaire was corrected and supplemented through the advice of an external expert who had extensive experience with the Delphi technique.

At the time of the drafting of the research background and questionnaire, e-mails were sent, in which the precautions to be taken by the subjects were delineated, including "prohibition of mutual exchange of opinions" and "self-administered questionnaire." The e-mails also contained the questionnaires, guidelines, and number of questionnaire surveys to be conducted. Ten days were spent for the distribution and retrieval of the first and second questionnaires, and 1 day was spent for the development of the second questionnaire.

\section{Analysis of the Results}

The panelists' opinions were gathered from the second questionnaire survey, and the stability of the survey was evaluated. When the interquartile range (IQR) was $<1$ and the coefficient of variation $(\mathrm{CV})$ was less than 0.5 , the survey was evaluated as stable. In addition, a portfolio analysis was performed, with the adequacy and feasibility indexes as the two axes. If more than two-thirds of the respondents submitted a "negative" (1 or 2 points), "having reservations" (3 points), or "affirmative" evaluation ( 4 or 5 points) for any one of the items, it was defined that consensus was reached on the corresponding question..$^{21-23)}$ If an item belonged to quartile I of the portfolio, it was denoted as having high adequacy and feasibility. However, if an item belonged to 
quartile III, it was denoted as having low adequacy and feasibility. MedCalc ver. 11.4 (MedCalc Software, Mariakerke, Belgium) was used for the statistical analysis.

\section{RESULTS}

\section{General Characteristics of the Panel}

The participating panel in the survey was composed of 10 members ( 3 men and 7 women), with a mean age of $41.2 \pm 6.3$ years. By occupation, the panel included 2 professors of family medicine, 1 professor of pediatrics and adolescent medicine, 2 professors of nutritional science, 1 professor of sports medicine, 1 professor of preventive medicine, 1 professor of sports physiology, and 2 health science researchers. The mean length of professional experience in their respective areas was $11 \pm 6.9$ years. The response rates for both the first and second surveys were $100.0 \%$ (Table 1 ).

Table 1. Respondents' demographic characteristics and response rate

\begin{tabular}{lcr}
\hline \multicolumn{1}{c}{ Characteristic } & $\begin{array}{c}\text { Completed round 1 } \\
(\mathrm{n}=10)\end{array}$ & $\begin{array}{r}\text { Completed round } 2 \\
(\mathrm{n}=10)\end{array}$ \\
\hline Sex & & \\
$\quad$ Male & $3(30.0)$ & $3(30.0)$ \\
$\quad$ Female & $7(70.0)$ & $7(70.0)$ \\
Age $(\mathrm{y})$ & $41.2 \pm 6.3$ & $41.2 \pm 6.3$ \\
Job & & \\
$\quad$ Professor of family medicine & $2(20.0)$ & $2(20.0)$ \\
Professor of pediatrics & $1(10.0)$ & $1(10.0)$ \\
$\quad$ Professor of nutrition & $2(20.0)$ & $2(20.0)$ \\
Professor of sports medicine & $1(10.0)$ & $1(10.0)$ \\
$\quad$ Professor of preventive medicine & $1(10.0)$ & $1(10.0)$ \\
Professor of exercise physiology & $1(10.0)$ & $1(10.0)$ \\
$\quad$ Researcher of health science & $2(20.0)$ & $2(20.0)$ \\
Career (y) & $11 \pm 6.9$ & $11 \pm 6.9$ \\
Response rate (\%) & 100 & 100
\end{tabular}

Values are presented as number (\%) or mean \pm standard deviation, unless otherwise indicated.

\section{Assessment of the Priority Population for Future Research}

The priority age groups to be considered at the time of designing the research on childhood obesity prevention were, in order of decreasing importance, "lower elementary school grades" (46 points), "higher elementary school grades" (43 points), "preschool” (26 points), "middle school" (24 points), and "high school" (11 points).

\section{Assessment of Intervention Methods for Future Research} The intervention methods that received an "affirmative" ( 4 or 5 points) consensus for their adequacy, according to age group, were "environmental control" for preschool children, and "dietary control," "physical activity control," and "environmental control" for elementary, middle, and high school students. Thus, an affirmative consensus was reached for $77.8 \%$ (7/9) of the items. Meanwhile, for preschool children, "dietary control" received a "having reservations" evaluation from 6 of the 10 panelists; and "physical activity control" received an "affirmative" evaluation from 6 of the 10 panelists. The IQR was $0-1$, and the CVs ranged from 0.06 to 0.18 , indicating that the survey was stable (Table 2).

\section{Assessment of Intervention Settings for Future Research} The intervention settings that received an affirmative consensus in terms of the anticipated effect size and feasibility for preschoolers were "home" and "kindergarten and childcare centers," while "community" received a consensus of "having reservations." The IQR was $0-1$, and the CVs ranged from 0.06 to 0.28 , indicating that the survey was stable. A consensus was reached for $83.3 \%$ of the items pertaining to the intervention setting (20/24) (Table 3).

In the portfolio analysis, "home" and "kindergarten and childcare centers" were the settings evaluated to have high effect sizes and feasibility (quartile I) for preschoolers. On the other hand, "primary care" and "community" settings were evaluated to have low effect sizes and feasibility (quartile III) for this age group. For elementary school students, "school" and "home" were evaluated as having high effect sizes and feasibility, while "primary care" and "community" were evaluated as having low effect sizes and feasibility. In the case of high school students, "school" was assessed as having a high effect size and feasibility,

Table 2. Results from round 2 of the survey: adequacy of intervention

\begin{tabular}{|c|c|c|c|c|c|c|c|}
\hline Intervention method & Median & Interquartile range & Coefficient of variation & Negative (\%) & Reservations (\%) & Positive (\%) & Adequacy index \\
\hline \multicolumn{8}{|l|}{ Preschool } \\
\hline Diet & 3 & 1 & 0.16 & 0 & 60 & 40 & 60.0 \\
\hline Physical activity & 4 & 1 & 0.18 & 0 & 40 & 60 & 67.5 \\
\hline Environment & 5 & 0.75 & 0.15 & 0 & 10 & 90 & 90.0 \\
\hline \multicolumn{8}{|l|}{ Elementary school } \\
\hline Diet & 4 & 0 & 0.17 & 0 & 20 & 80 & 75.0 \\
\hline Physical activity & 5 & 0.75 & 0.10 & 0 & 0 & 100 & 92.5 \\
\hline Environment & 5 & 0.75 & 0.10 & 0 & 0 & 100 & 92.5 \\
\hline \multicolumn{8}{|l|}{ Middle/high school } \\
\hline Diet & 5 & 0 & 0.14 & 0 & 10 & 90 & 92.5 \\
\hline Physical activity & 5 & 0 & 0.06 & 0 & 0 & 100 & 97.5 \\
\hline Environment & 5 & 0 & 0.09 & 0 & 0 & 100 & 95.0 \\
\hline
\end{tabular}


Table 3. Results from round 2 of the survey: effectiveness/feasibility of the intervention according to setting

\begin{tabular}{|c|c|c|c|c|c|c|c|}
\hline Setting & Interquartile range & Coefficient of variation & Negative (\%) & Reservations (\%) & Positive (\%) & Effectiveness index & Feasibility index \\
\hline \multicolumn{8}{|l|}{ Preschool } \\
\hline Child care & $1 / 1$ & $0.16 / 0.16$ & $0 / 0$ & $10 / 10$ & $90 / 90$ & 87.5 & 82.5 \\
\hline Home & $0 / 1$ & $0.09 / 0.16$ & $0 / 0$ & $0 / 10$ & $100 / 90$ & 95 & 87.5 \\
\hline Primary care & $1 / 0$ & $0.21 / 0.15$ & $50 / 20$ & $50 / 80$ & $0 / 0$ & 37.5 & 45 \\
\hline Community & $0.10 / 0.05$ & $0.13 / 0.18$ & $0 / 10$ & $80 / 70$ & $20 / 20$ & 55 & 52.5 \\
\hline \multicolumn{8}{|c|}{ Elementary school } \\
\hline School & $0 / 0$ & $0.06 / 0.09$ & $0 / 0$ & $0 / 0$ & $100 / 100$ & 97.5 & 95 \\
\hline Home & $1 / 1$ & $0.12 / 0.12$ & $0 / 0$ & $0 / 0$ & $100 / 100$ & 87.5 & 85 \\
\hline Primary care & $0 / 0.65$ & $0.16 / 0.15$ & $10 / 0$ & $80 / 70$ & $10 / 30$ & 50 & 57.5 \\
\hline Community & $0.15 / 0.75$ & $0.20 / 0.13$ & $0 / 0$ & $80 / 30$ & $20 / 70$ & 57.5 & 67.5 \\
\hline \multicolumn{8}{|c|}{ Middle/high school } \\
\hline School & $1 / 1$ & $0.11 / 0.16$ & $0 / 0$ & $0 / 10$ & $100 / 90$ & 90 & 82.5 \\
\hline Home & $0.70 / 0.70$ & $0.21 / 0.21$ & $0 / 0$ & $70 / 70$ & $30 / 30$ & 60 & 60 \\
\hline Primary care & $1 / 1$ & $0.21 / 0.28$ & $10 / 20$ & $40 / 30$ & $50 / 50$ & 60 & 60 \\
\hline Community & $0.75 / 1$ & $0.22 / 0.25$ & $10 / 20$ & $20 / 40$ & $70 / 40$ & 67.5 & 55 \\
\hline
\end{tabular}

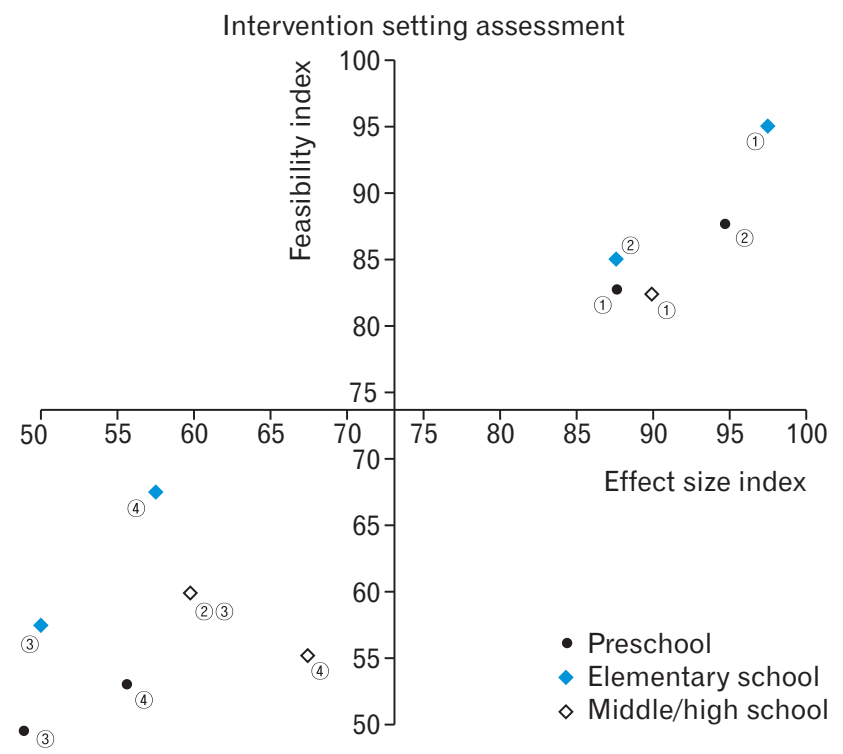

Figure 1. Portfolio: feasibility and effect-size indices of suggested intervention settings for children in preschool, elementary school, and middle/high school. (1) Childcare or school; (2) home; (3) primary care; (4) community.

while "home," "primary care," and "community" were assessed as having low effect sizes and feasibility (Figure 1).

\section{Assessment of Intervention Outcomes for Future \\ Research}

The panelists were also asked to evaluate key outcome measures of obesity interventions, including anthropometric indexes such as "body mass index (BMI)," "BMI percentile," "relative weight on the standard weight for each sex and age group," "prevalence of obesity in the group," “waist circumference," "skin thickness," and "body fat." In the portfolio analysis, "BMI percentile" was deemed as having high adequacy and feasibility for preschoolers. In the case of the elementary
Anthropometric outcome assessment

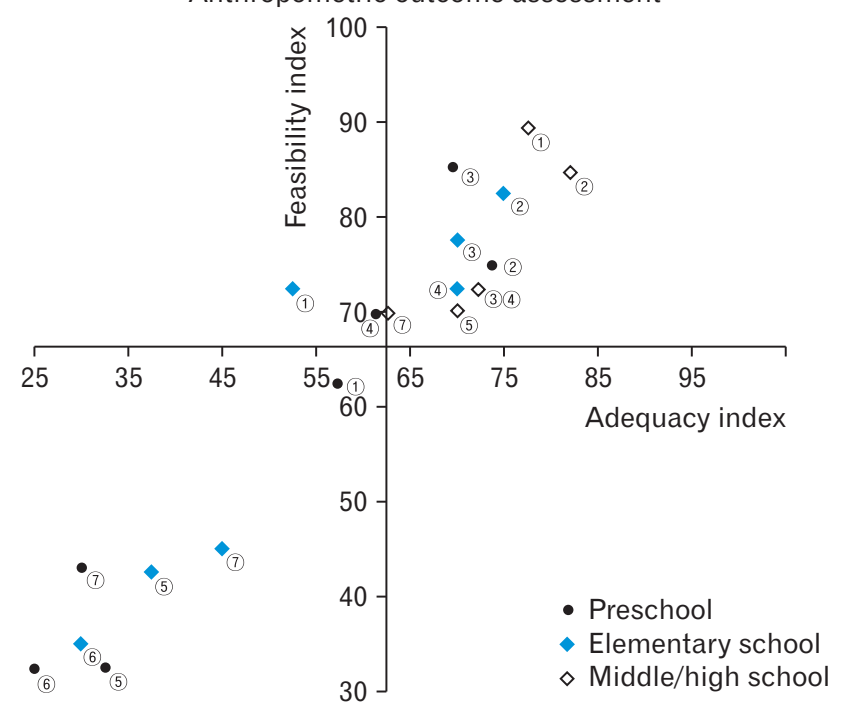

Figure 2. Portfolio: adequacy and feasibility indices of suggested anthropometric outcomes for children in preschool, elementary school, and middle/high school. (1) BMI; (2) BMI percentile; (3) relative body weight; (4) prevalence of obesity; (5) waist circumference; (6) skinfold thickness; (7) body fat. BMl, body mass index.

school students, "BMI percentile," "relative weight on the standard weight for each sex and age group," and "prevalence of obesity in the group" were the indexes with high adequacy and feasibility. For the middle and high school students, the indexes evaluated as having high adequacy and feasibility included "BMI," "BMI percentile," "relative weight on the standard weight for each sex and age group," "prevalence of obesity of the group," and "waist circumference" (Figure 2).

In the portfolio analysis of clinical indexes such as "blood pressure," "serum glucose level," "cholesterol," "liver function," and "proteinuria," all the indexes were assessed as having low adequacy and feasibility for both preschoolers and elementary school students. For the middle and high school students, "blood pressure," "serum glucose level," and 
"cholesterol" were the indexes scored with high levels of adequacy and feasibility (Figure 3).

In the portfolio analysis for the measurement of intermediate variables, including "knowledge of, attitudes toward, and beliefs about nutrition," "eating-related behavior," "quantity of food eaten," "screen time," "amount and intensity of exercise," and "emotions such as depression and sense of self-efficacy," the indexes assessed as having high adequacy but low feasibility were "knowledge of, attitudes toward, and beliefs about nutrition," and "eating-related behavior" for preschoolers. For elementary school students, all the indexes except "emotions such as depression and sense of self-efficacy" were scored as having high adequacy and feasibility. In the case of middle and high school students, all the intermediate variables except "knowledge of, attitudes toward, and beliefs about nutrition" were evaluated as having high adequacy and feasibility (Figure 4).

\section{Assessment of Intervention Durations for Future Research}

The periods deemed appropriate for the assessment of the intervention outcomes were 9 months for the "anthropometric indexes" (mean \pm standard deviation score: $2.5 \pm 0.53$ ), 1 year for the "clinical indexes" (2.9 \pm 0.32$)$, and 6 months for the "intermediate variables" $(2.1 \pm 0.32)$. The IQR was $0-1$, and the CVs ranged from 0.11 to 0.21 , indicating that the survey was stable.

\section{DISCUSSION}

In this study, a Delphi survey was conducted to examine the priority age group for the research on childhood obesity prevention, and the adequacy and feasibility of different intervention methods. It is signifi-

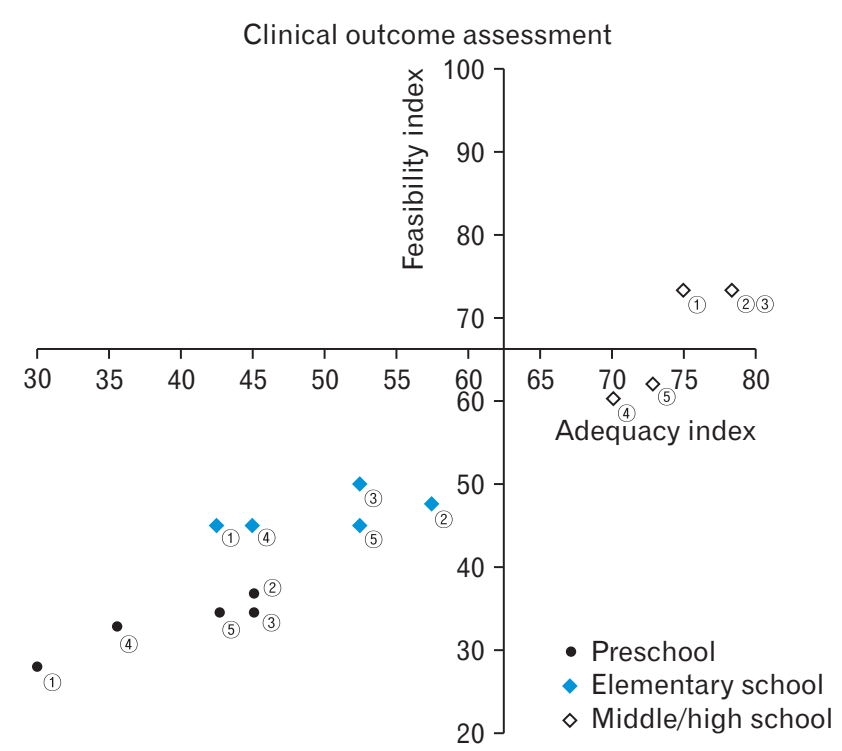

Figure 3. Portfolio: adequacy and feasibility indices of suggested clinical outcomes for children in preschool, elementary school, and middle/high school. (1) Blood pressure; (2) serum glucose; (3) cholesterol; (4) liver function; (5) proteinuria. cant that the Delphi technique was applied for the first time in Korea to assess the priorities and intervention methods for a future research on childhood obesity prevention.

Elementary school students were judged as the highest priority population for the research on childhood obesity prevention. For preschoolers, "kindergarten and childcare centers" and "home" were selected as the priority settings, and "environmental control" interventions that measure "BMI percentile" were found as having high adequacy and feasibility among the various methods and outcome measures. In the case of the elementary school students, the priority settings for future research were established as "school" and "home," and high adequacy and feasibility were assigned to interventions focused on "dietary control," "physical activity control," and "environmental control" and measuring "BMI percentile," "relative weight on the standard weight for each sex and age group," "prevalence of obesity of the group," "nutritional knowledge, attitudes, and beliefs," "eating-related behavior," "quantity of food eaten," "screen time," and "amount and intensity of exercise." For middle and high school students, "school" was selected as the priority setting, and interventions that focused on "dietary control," "physical activity control," and "environmental control" and measured most of the possible indicators were assessed as having high adequacy and feasibility.

The priority age groups of the research on childhood obesity prevention were (in decreasing order) "elementary school," "preschool," and "middle and high school" students. In the results of the questionnaire survey conducted by Wu et al. ${ }^{24)}$ about the prevention of childhood obesity, the priority age group was "preschool students," followed by "elementary school students." When anticipating the outcomes of the research on childhood obesity prevention in a domestic

Intermediate outcome assessment

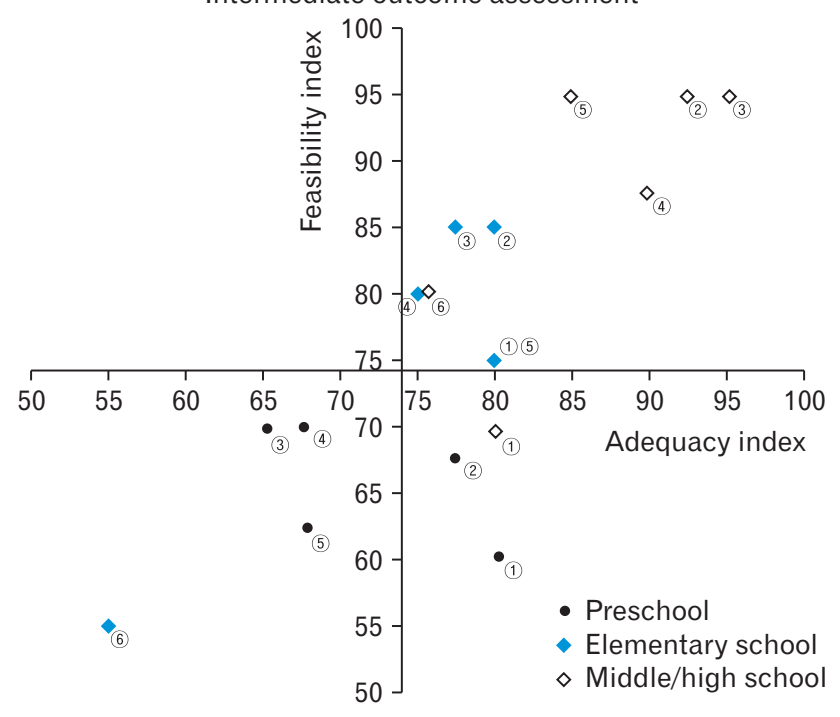

Figure 4. Portfolio: adequacy and feasibility indices of suggested intermediate outcomes for children in preschool, elementary school, and middle/high school. (1) Nutritional knowledge, attitudes, and beliefs; (2) eating-related behavior; (3) quantity of food eaten; (4) screening time; (5) amount and intensity of exercise; (6) psychological factors such as depression and sense of self-efficacy. 
environment, the panelists of this study considered elementary school students to be the highest-priority group. This may be because elementary school students do not yet have fixed lifestyles and are at an age when an intervention would be realistic owing to the low burden of their school studies. In addition, elementary school students may be the most effective target population for the prevention of obesity or related diseases.

Regarding the appropriate intervention method(s) for the research on childhood obesity prevention for each age group, "environmental control" was assessed as appropriate for preschoolers, and "environmental control," "physical activity control," and "dietary control" were deemed appropriate for elementary, middle, and high school students. In our assessment, this could be because meals and physical activities are dictated by the surrounding environment without the students' selection or control, and because preschoolers lack self-control. In a meta-analysis of 37 obesity intervention trials conducted over a period of 12 weeks in adolescents aged $<18$ years, BMI was reduced by $0.11 \mathrm{~kg} / \mathrm{m}^{2}(\mathrm{P}=0.02)$ when only "physical activity control" was applied and by $0.12 \mathrm{~kg} / \mathrm{m}^{2}(\mathrm{P}=0.16)$ when only "dietary control" was applied. However, when interventions such as "physical activity control" and "dietary control" were combined, BMI was reduced by $0.18 \mathrm{~kg} / \mathrm{m}^{2}$ $(\mathrm{P}<0.0001) .{ }^{25}$ Considering that the combined intervention was more effective than the respective individual interventions, combined interventions could achieve much better effects among elementary school students, and middle and high school students.

We assessed both, the anticipated effect size and feasibility of different settings for research on childhood obesity prevention for each age group through a portfolio analysis. As we anticipated that high effect sizes and feasibilities could be achieved through interventions at "kindergarten and childcare centers" and "home" for preschoolers, "school" and "home" for elementary school students, and "school" for middle and high school students, we deemed that these intervention settings should be highly prioritized. On the other hand, the intervention effects and feasibilities were anticipated to be low for the "primary care" and "community" settings for preschoolers and elementary school students, and for the "home," "primary care," and "community" settings for middle and high school students. Thus, the specific obstacles in these settings should be examined. Primary care settings are thought to be more appropriate for treatment interventions than for preventive interventions. "Home" received a reserved assessment for middle and high school students, likely because the time such students spend at home is short, as most of their time is spent at school or in private academies.

A diverse range of outcome measures have been used in research studies on childhood obesity prevention in Korea. However, systematic comparison of these studies has been difficult because most of the researchers arbitrarily used cross-sectional self-defined outcome measures. ${ }^{26-28)}$ Thus, in this study, we identified the most appropriate and feasible indexes among those used previously, in an effort to standardize the tools for measuring outcomes. The number of available measurement tools increased as the age group increased in the case of the anthropometric indexes. In the case of the clinical indexes, all were assigned low adequacy and feasibility for preschoolers and elementary school students, while indexes such as "blood pressure," "serum glucose level," and "cholesterol" were deemed to have high adequacy and feasibility for use among middle and high school students. Regarding the anthropometric or intermediate indexes that influenced the clinical indexes, "quantity of food eaten," "screen time," and "amount and intensity of exercise" cannot be considered as age-appropriate indexes for preschoolers. For elementary school students, the assignment of low adequacy and feasibility to "emotions such as depression and sense of self-efficacy" needs to be reassessed.

The durations selected as appropriate for the assessment of outcomes of research on childhood obesity prevention were 9 months, 1 year, and 6 months for the anthropometric indexes, clinical indexes, and intermediate variables, respectively. The results of an evidencebased research review suggested that proper assessment can be made only if school-based interventions are executed for a minimum of 6 months and if community-based interventions are executed for a minimum of 1 year. ${ }^{19,20)}$

The limitations of this study include the following: First, because a questionnaire with structuralized and closed questions was used for the first survey, the panel members' ability to express their opinions might have been restricted. Second, the results might have been exaggerated or downsized, as weighting was applied during the calculation of the importance scores, adequacy indexes, and feasibility indexes used for the assessment of priorities.

In this study, the priorities for designing the research on childhood obesity prevention were assessed with the Delphi technique, and their adequacies and feasibilities were considered. The highest-priority population for the research on childhood obesity prevention was elementary school students. Different settings, contents, assessment methods, and durations were anticipated to be effective in the different age groups. Accordingly, future research is needed to evaluate intervention outcomes by developing a research on childhood obesity prevention by using appropriate indexes over a prolonged period, with consideration of the populations and settings with high levels of adequacy and feasibility.

\section{CONFLICT OF INTEREST}

No potential conflict of interest relevant to this article was reported.

\section{ACKNOWLEDGMENTS}

This research was supported by a fund from the research of Korea Centers for Disease Control and Prevention (2015-ER6402-00).

\section{REFERENCES}

1. World Health Organization. Fact sheets: obesity and overweight [Internet]. Geneva: World Health Organization; 2016 [cited 2016 Feb 11]. 
Available from: http://www.who.int/mediacentre/factsheets/fs311/ en/.

2. Strauss RS, Pollack HA. Epidemic increase in childhood overweight, 1986-1998. JAMA 2001;286:2845-8.

3. Ministry of Education. 2014 School health survey: results of sample survey [Internet]. Sejong: Ministry of Education; 2014 [cited 2016 Feb 11]. Available from: http://www.moe.go.kr/boardCnts/view.do?boardI $\mathrm{D}=348 \& \mathrm{lev}=0$ \&statusYN=W\&s=moe\&m=040103\&opType=N\&boardS eq $=60946$.

4. Zametkin AJ, Zoon CK, Klein HW, Munson S. Psychiatric aspects of child and adolescent obesity: a review of the past 10 years. J Am Acad Child Adolesc Psychiatry 2004;43:134-50.

5. Ford ES, Moriarty DG, Zack MM, Mokdad AH, Chapman DP. Self-reported body mass index and health-related quality of life: findings from the Behavioral Risk Factor Surveillance System. Obes Res 2001;9:21-31.

6. Schwimmer JB, Burwinkle TM, Varni JW. Health-related quality of life of severely obese children and adolescents. JAMA 2003;289:1813-9.

7. Jung YH, Ko S, Lim HJ. The socioeconomic cost of adolescent obesity. Health Soc Welf Rev [Internet]. 2010 [cited 2016 Feb 11];30:195-219. Available from: http://repository.kihasa.re.kr:8080/handle/201002/5629.

8. Magarey AM, Daniels LA, Boulton TJ, Cockington RA. Predicting obesity in early adulthood from childhood and parental obesity. Int J Obes Relat Metab Disord 2003;27:505-13.

9. Guo SS, Wu W, Chumlea WC, Roche AF. Predicting overweight and obesity in adulthood from body mass index values in childhood and adolescence. Am J Clin Nutr 2002;76:653-8.

10. Kim MS, Choi MS, Kim KN. Effect of nutrition education and exercise intervention on physical and dietary patterns of some obese children. Korean J Community Nutr 2011;16:426-38.

11. Cho YG, Song HR, Kim KA, Kang JH, Kang JH, Song YH, et al. Effect of a school-based intervention for overweight children "fitness class" performed on elementary schools located in Seoul. Korean J Obes 2009;18:146-57.

12. Yun SJ, Lee K. Effects of a school-based obesity prevention program in adolescents. J Korean Acad Fam Med 2008;29:585-94.

13. Lee K, Nam HM, Lee JR. Effect of elementary school based intervention to prevent obesity in children. Korean J Obes 2007;16:125-9.

14. Seo NS, Kim YH, Kang HY. Effects of an obesity control program based on behavior modification and self-efficacy in obese elementary school children. J Korean Acad Nurs 2005;35:611-20.

15. Linstone HA. The Delphi method: techniques and applications by
Harold A. Linstone. Am Mark Assoc 1976;13:317-8.

16. Rho SY. Delphi technique: future forecasting with professional insights. Plan Policy 2006;(299):53-62.

17. Lee YK, Shin ES, Shim JY, Min KJ, Kim JM, Lee SH, et al. Developing a scoring guide for the appraisal of guidelines for research and evaluation II instrument in Korea: a modified Delphi consensus process. J Korean Med Sci 2013;28:190-4.

18. Park JH, Lee R, Sohn I. Setting school-level educational goal and objectives with the modified Delphi method. Korean J Med Educ 2011;23:243-52.

19. Wang Y, Cai L, Wu Y, Wilson RF, Weston C, Fawole O, et al. What childhood obesity prevention programmes work?: a systematic review and meta-analysis. Obes Rev 2015;16:547-65.

20. Wang Y, Wu Y, Wilson RF, Bleich S, Cheskin L, Weston C, et al. Childhood obesity prevention programs: comparative effectiveness review and meta-analysis. Rockville (MD): Agency for Healthcare Research and Quality; 2013.

21. Khang YH, Yoon SJ, Kang GW, Kim CY, Yoo KY, Shin YS. An application of Delphi method to the assessment of current status of cancer research. Korean J Prev Med 1998;31:844-56.

22. Khorramshahgol R, Moustakis VS. Delphic hierarchy process (DHP): a methodology for priority setting derived from the Delphi method and analytical hierarchy process. Eur J Oper Res 1988;37:347-54.

23. Hwang SW, Gwon SH, Lee MK. A study on the Delphi survey on R\&D projects for urban regeneration in Korea. Proceedings of the Korea Technology Innovation Society Conference; 2009; Daejeon, Korea. Seoul: Korea Technology Innovation Society; 2009.

24. Wu Y, Lau BD, Bleich S, Cheskin L, Boult C, Segal JB, et al. Future research needs for childhood obesity prevention programs. Rockville (MD): Agency for Healthcare Research and Quality (US); 2013.

25. Waters E, de Silva-Sanigorski A, Hall BJ, Brown T, Campbell KJ, Gao Y, et al. Interventions for preventing obesity in children. Cochrane Database Syst Rev 2011;(12):CD001871.

26. Sung KS, Yoon YM, Kim EJ. Meta-analysis of the effects of obesity management program for children. Child Health Nurs Res 2013;19:262-9.

27. Cai L, Wu Y, Wilson RF, Segal JB, Kim MT, Wang Y. Effect of childhood obesity prevention programs on blood pressure: a systematic review and meta-analysis. Circulation 2014;129:1832-9.

28. De Bourdeaudhuij I, Van Cauwenberghe E, Spittaels H, Oppert JM, Rostami C, Brug J, et al. School-based interventions promoting both physical activity and healthy eating in Europe: a systematic review within the HOPE project. Obes Rev 2011;12:205-16. 